\title{
THE FIRST AND SECOND VARIATIONS OF A DOUBLE INTEGRAL FOR THE CASE OF VARIABLE LIMITS*
}

BY

H. A. SIMMONS

\section{INTRODUCTION}

Among the many interesting general problems in the calculus of variations is the following one. Suppose we have given the double integral

$$
I=\iint_{A_{0}} f(x, y, z, p, q) d x d y
$$

where $A_{0}$ is the area in the $x y$-plane over which the integral is taken (see Figure 1), and where $p \equiv \partial z / \partial x$, $q \equiv \partial z / \partial y$, and suppose we also have given a fixed surface $\varphi(x, y, z)=0$ which is arbitrary. It is then required to find among all surfaces which are representable in the form $z=z(x, y)$ and which have their edges on the fixed surface $\varphi=0$ that one which minimizes the double integral $I$.

In studying the problem just stated, one immediately finds need of the first and second variations of the integral $I$. In obtaining them, it is desirable to

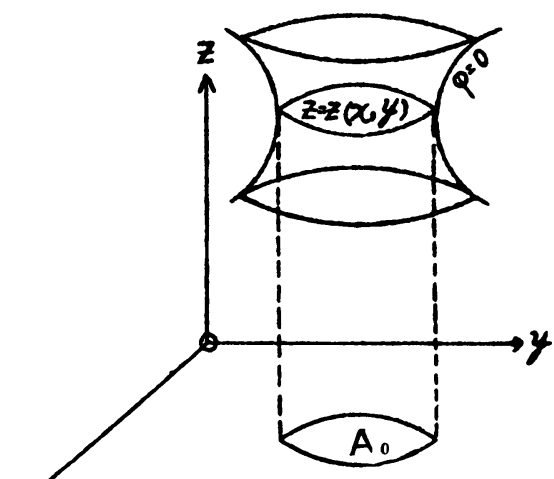

Fig. 1 assume that the sought surface $z=z(x, y)$ has already been found and then to consider a one-parameter family of surfaces of the type

$$
z=z(x, y)+a \zeta(x, y)
$$

containing the surface $z=z(x, y)$ for $a=0$. We allow $\zeta$ to be an arbitrary function of $x$ and $y$, but we take the value of the integral only on the portion of each surface of the family which is bounded by the fixed surface $\varphi=0$ (see Figure 1). When $z$ in the integrand of the double integral $I$ is replaced

* Presented to the Society, April 2, 1926; received by the editors before October, 1925. 
by $z+a \zeta, I$ becomes a function of $a$ and of course the limits of the integral also change. We may then write in place of the integral above

$$
I(a)=\iint_{A_{a}} f\left(x, y, z+a \zeta, p+a \zeta_{x}, q+a \zeta_{y}\right) d x d y,
$$

where $A_{a}$ is the area bounded by the projection on the $x y$-plane of the curve of intersection of the surface $\varphi=0$ with that surface of the family $z=z(x, y)+a \zeta(x, y)$ whose parameter value is $a$. Proceeding in this manner and then differentiating $I(a)$ with respect to $a$ and putting $a=0$, we obtain the two derivatives $I^{\prime}(0), I^{\prime \prime}(0)$, which are of great service in studying the problem.

Heretofore, only the first derivative, ${ }^{*} I^{\prime}(0)$, has been discussed in detail for problems of this type for which the boundary of the surfaces $z=z(x, y)$ over which the integral $I$ is taken is allowed to vary arbitrarily on a fixed surface $\varphi=0$. The expression for $I^{\prime}(0)$ presented in this paper seems to be the most satisfactory one that has been obtained, the results of other writers, Sarrus for example, being unsymmetric and very difficult to apply. No one, so far as is known to the author, has computed the second derivative $I^{\prime \prime}(0)$ heretofore, apparently because of the very great complications which have arisen when the computation has been attempted. These difficulties have been described by Bolza $\dagger$ and mention of them has also been made in the French Encyclopédie.t

It is the purpose of this paper to compute symmetric, usable forms for $I^{\prime}(0)$ and $I^{\prime \prime}(0)$, especially $I^{\prime \prime}(0)$. The author believes that those who study the method used here, which was suggested by Professor G. A. Bliss, will agree that it is a much better mode of attack than has been used heretofore. Furthermore, the reader will without doubt appreciate the relative simplicity and the perfect symmetry of the results if he compares them with those obtainable, let us say, by the method of Sarrus.

In $\$ 1$ a theorem is proved concerning the differentiation of a double integral with respect to a parameter which occurs both in the integrand and in the limits of integration. In $\$ 2$ the problem of the following sections is stated in a more precise form than is given above. In $\$ 3$ the first derivative $I^{\prime}(0)$ is computed and from the new expression for it some results are deduced which have already been obtained by other methods. In $\$ 4$, we compute $I^{\prime \prime}(0)$. It is found to be equal to a double integral with the same integrand as that which appears for the corresponding problem for which

\footnotetext{
* See Sarrus's paper in Mémoires, Savants Etrangers, vol. 10 (1866) (Prize article).

† Vorlesungen über Variationsrechnung, p. 669.

‡ Encyclopédie des Sciences Mathématiques, tome II, vol. 6, p. 166.
} 
the limits are fixed, plus a line integral which involves $\zeta^{2}$ but no other power or derivative of $\zeta$. In $\S 5$, we state a new necessary condition for a minimum of the integral $I$. The formulation of the statement is in terms of a boundary value problem associated with the second variation $I^{\prime \prime}(0)$. In $\S 6$, finally, applications of two of our formulas are made to the case where $z=z(x, y)$ is a surface of minimum area.

The author is pleased to acknowledge the assistance of Professor G. A. Bliss, who suggested the problem studied in this paper. It was through his careful direction and inspiration, for which the author is very thankful, that this paper was written.

1. The derivatives of a double integral with respect to a parameter. Let $C_{0}$ be a simply closed curve with equations

$$
x=\xi(u), \quad y=\eta(u),
$$

where $\xi, \eta$ are defined for all real values of $u$, are of class $C^{\prime \prime},{ }^{*}$ have $\xi^{\prime 2}+\eta^{\prime 2}=1$, and have a period $l$ equal to the length of $C_{0}$.

Introduce near $C_{0}$ a $u v$-coördinate system determined by the equations

$$
x=\xi(u)+v \eta^{\prime}(u), \quad y=\eta(u)-v \xi^{\prime}(u) .
$$

These equations define a unique pair of coördinates $(u, v)$ for each point $(x, y)$ near $C_{0}$, since for each pair of values $(x, y)$ on $C_{0}$ they define a unique pair $(u, v)=(u, 0)(0 \leqq u \leqq l)$ and since along $C_{0}$ the functional determinant

$$
\left|\begin{array}{ll}
x_{u} & y_{u} \\
x_{v} & y_{v}
\end{array}\right|=\left|\begin{array}{lr}
\xi^{\prime}+v \eta^{\prime \prime} & \eta^{\prime}-v \xi^{\prime \prime} \\
\eta^{\prime} & -\xi^{\prime}
\end{array}\right|=-(1+v / \rho)
$$

is different from zero. $\dagger$ Literal subscripts to functions, here as in all of this paper, indicate partial differentiation with respect to the letter written; and in the last formula $\rho$ is the radius of curvature of $C_{0}$. The $u$-curves are the parallel curves to $C_{0}$ and the $v$-curves are the normals to $C_{0}$, thus establishing near $C_{0}$ a curvilinear coördinate system $u, v$ with orientation opposite to that of $x, y$.

Now consider a family of curves, one of which, $C_{a}$ (see Figure 2) is given by the equations

$$
x=\xi(u)+v(u, a) \eta^{\prime}(u), \quad y=\eta(u)-v(u, a) \xi^{\prime}(u),
$$

- A function $f(x)$ is of class $C^{(n)}$ on the interval $x_{0} \leqq x \leqq x_{1}$ if its $n$th derivative is continuous at every point of that interval.

$\dagger$ For details as to the neighborhood in which the correspondence is unique, see Bliss's Princeton Colloquium Lectures, 1913, p. 20. 
where $v(u, a)$ is defined and of class $C^{\prime \prime}$ for all $(u, a)$ having $u$ real and $a$ sufficiently near zero; where $v(u, a)$ has for the variable $u$ a period $L(a)$ reducing to $L(0)=l$ when $a=0$; and where $v(u, 0) \equiv 0$. All of the curves $C_{a}$ are closed on account of this periodic property, and each $C_{a}$ is further simply closed for all values of $a$ sufficiently near zero since $C_{0}$ is simply closed. We let $A_{a}$ denote the area bounded by $C_{a}$.

Let $C_{1}$ be the curve defined by (1) when the variable $v$ is replaced by a special



Fig. 2 constant value $v_{1}<0$, and designate by $A_{1}$ the area bounded by $C_{1}$ (see Figure 2 above). In the paragraphs below, $v_{1}$ is supposed to be as near zero as may be required in order that the curve $C_{1}$ defined by the constant value $v_{1}$ shall lie in the neighborhood of $C_{0}$ in which the $u v$-coördinate system described above is well-defined.

Let $g(x, y, a)$ be a function of $x, y, a$ which is of class $C^{\prime \prime}$ for all sets $(x, y, a)$ having $(x, y)$ in a neighborhood of the area $A_{0}$ bounded by $C_{0}$, and having $a$ sufficiently near $a=0$. Let us define $J(a)$ by the formula

$$
J(a) \equiv \iint_{A_{a}} g(x, y, a) d x d y .
$$

We wish to find the derivatives $J^{\prime}(0)$ and $J^{\prime \prime}(0)$ of this integral. It is convenient in order to make these differentiations to have $J(a)$, (4), written in the form

$$
J(a)=\iint_{A_{1}} g(x, y, a) d x d y+\iint_{\Delta A} g(x, y, a) d x d y,
$$

where $A_{1}$ is as defined above and $\Delta A$ is the area bounded by the curves $C_{1}$ and $C_{a}$ (see Figure 2). The derivative of the first integral of $\left(4^{\prime}\right)$ has, as is well known, the value

$$
\iint_{A_{1}} g_{a}(x, y, a) d x d y .
$$

To find the derivative of the second integral in $\left(4^{\prime}\right)$, we first transform it to the $u v$-coördinate system by means of (1), remembering the value (2) of the functional determinant $x_{u} y_{v}-x_{v} y_{u}$, and obtain

$$
\iint_{\Delta A} g(x, y, a) d x d y=\int_{0}^{l}\left[\int_{v_{1}}^{v(u, a)} g\left(\xi+v \eta^{\prime}, \eta-v \xi^{\prime}, a\right)(1+v / \rho) d v\right] d u .
$$


The derivative of the last written integral is obtainable by the usual formula for the derivative of a simple integral containing a parameter. Since the parameter $a$ occurs only in the upper limit of the inner integral and explicitly in $g$, we find for this derivative

$$
\int_{0}^{l} v_{a} g(1+v / \rho) d u+\int_{0}^{l} \int_{v_{1}}^{v(u, a)} g_{a}(1+v / \rho) d v d u,
$$

where in the first integral the $v$, wherever it occurs, is the function $v(u, a)$. Adding this result to the expression (5), we obtain

$$
\begin{aligned}
J^{\prime}(a)=\iint_{A_{1}} g_{a} d x d y+\int_{0}^{l} \int_{v_{1}}^{v(u, a)} g_{a}(1 & +v / \rho) d v d u \\
& +\int_{0}^{l} v_{a} g\left(\xi^{\prime}+v \eta^{\prime}, \eta-v \xi^{\prime}, a\right)(1+v / \rho) d u
\end{aligned}
$$

or, after transforming the second integral to $x y$-coördinates again,

$$
J^{\prime}(a)=\iint_{A_{a}} g_{a} d x d y+\int_{0}^{l} v_{a} g\left(\xi+v \eta^{\prime}, \eta-v \xi^{\prime}, a\right)(1+v / \rho) d u .
$$

To calculate the value of the second derivative $J^{\prime \prime}(a)$, we can in like manner find the derivative of the first term of $(7)$ to be

$$
\iint_{A_{a}} g_{a a} d x d y+\int_{0}^{l} g_{a} v_{a}(1+v / \rho) d u .
$$

By the formula for differentiating a simple integral with respect to a parameter, the derivative of the last term of (7) is

Therefore

$$
\int_{0}^{l}\left[\left(g v_{a a}+g_{v} v_{a}^{2}+g_{a} v_{a}\right)(1+v / \rho)+g v_{a}^{2} / \rho\right] d u .
$$

$$
J^{\prime \prime}(a)=\iint_{A_{a}} g_{a a} d x d y+\int_{0}^{l}\left[\left(g v_{a a}+2 g_{a} v_{a}+g_{v} v_{a}^{2}\right)(1+v / \rho)+g v_{a}^{2} / \rho\right] d u .
$$

Putting $a=0$ in (7) and (8), we obtain the desired results which are described in the following theorem.

Theorem 1. The derivatives $J^{\prime}(0)$ and $J^{\prime \prime}(0)$ of the function $J(a)$, defined by the double integral (4), taken over the region $A_{a}$ bounded by the curve $C_{a}$, defined by the equations (3), have the values

$$
\begin{aligned}
& J^{\prime}(0)=\iint_{A_{0}} g_{a} d x d y+\int_{0}^{l} g v_{a} d u, \\
& J^{\prime \prime}(0)=\iint_{A_{0}} g_{a a} d x d y+\int_{0}^{l}\left(g v_{a a}+g v_{a}^{2} / \rho+2 g_{a} v_{a}+g_{v} v_{a}^{2} / \rho\right) d u .
\end{aligned}
$$


Although these derivatives (9) have been computed for a one-parameter family of curves of the special type (3), we can obtain from them analogous formulas for a more general family of the form

$$
x=X(\tau, a), \quad y=Y(\tau, a) .
$$

We suppose that (10) represents a one-parameter family of simply closed curves containing $C_{0}$ for $a=0$ and having $\tau$ as length of $\operatorname{arc}$ on $C_{0}$. The functions $X, Y$ are of class $C^{\prime \prime}$ for all values of $(\tau, a)$ having $\tau$ real and the values of $a$ sufficiently near zero. They have a period $T(a)$ for every $a$ with $T(0)=l$, the length of $C_{0}$. Such a family is always representable in the form (3) above by solving the equations

$$
\xi(u)+v \eta^{\prime}(u)-X(\tau, a)=0, \eta(u)-v \xi^{\prime}(u)-Y(\tau, a)=0
$$

for $v$ and $\tau$ as functions of $u$ and $a$. According to the implicit function theorem referred to above, this can always be done since equations (11) have the particular set of solutions $(v, \tau, u, a)=(0, u, u, 0)$ for $0 \leqq u \leqq l$, on which

$$
\left|\begin{array}{rr}
\eta^{\prime} & -X_{\tau} \\
-\xi^{\prime} & -Y_{\tau}
\end{array}\right|=\left|\begin{array}{rr}
\eta^{\prime} & -\xi^{\prime} \\
-\xi^{\prime} & -\eta^{\prime}
\end{array}\right|=-1 .
$$

Hence, according to the implicit function theorem referred to above, if $J(a)$ is the value of $J$ on the region $A_{a}$ bounded by the curve $C_{a}$, defined by (10), its derivatives $J^{\prime}(0)$ and $J^{\prime \prime}(0)$ are given by (9), where $v_{a}$ is obtained by differentiating equations (11) with respect to $a$ and solving the resulting equations for $v_{a}, \tau_{a}$; and where $v_{a a}$ is similarly obtained after a second differentiation and solution of the two resulting equations, which are linear in $v_{a a}$ and $\tau_{a a}$, for $v_{a a}$. The derivatives are

$$
\begin{gathered}
X_{\tau} \tau_{a}-\eta^{\prime} v_{a}+X_{a}=0 ; \quad Y_{\tau} \tau_{a}+\xi^{\prime} v_{a}+Y_{a}=0 ; \\
X_{\tau} \tau_{a a}-\eta^{\prime} v_{a a}+X_{\tau \tau} \tau_{a}^{2}+2 X_{a \tau} \tau_{a}+X_{a a}=0 ; \\
Y_{\tau} \tau_{a a}+\xi^{\prime} v_{a a}+Y_{\tau \tau} \tau_{a}^{2}+2 Y_{a \tau} \tau_{a}+Y_{a a}=0 .
\end{gathered}
$$

From the two equations in the first line of (12) we obtain $v_{a}$ and $\tau_{a}$. Then using the value found for $\tau_{a}$, namely

$$
\tau_{a}=\frac{-\xi^{\prime} X_{a}-\eta^{\prime} Y_{a}}{\xi^{\prime} X_{\tau}+\eta^{\prime} Y_{\tau}}=-X_{\tau} X_{a}-Y_{\tau} Y_{a},
$$

in the last two equations, we solve them, as stated above, for $v_{a a}$. This gives for $v_{a}$ and $v_{a a}$ the values

$$
\begin{aligned}
v_{a}= & X_{a} Y_{\tau}-X_{\tau} Y_{a} \\
v_{a a}= & \left(X_{\tau \tau} Y_{\tau}-X_{\tau} Y_{\tau \tau}\right)\left(X_{\tau} X_{a}+Y_{\tau} Y_{a}\right)^{2} \\
& -2\left(X_{a \tau} Y_{\tau}-X_{\tau} Y_{a \tau}\right)\left(X_{\tau} X_{a}+Y_{\tau} Y_{a}\right)+X_{a a} Y_{\tau}-X_{\tau} Y_{a a} .
\end{aligned}
$$


Substituting the values (13) of $v_{a}$ and $v_{a a}$ in equations (9), we obtain the more general result desired, which we express in the

Corollary. The derivatives $J^{\prime}(0)$ and $J^{\prime \prime}(0)$ of the double integral (4), taken over the region $A_{a}$, bounded by the curve $C_{a}$ defined by the equations (10), have the values

$$
\begin{aligned}
J^{\prime}(0) & =\iint_{A_{0}} g_{a} d x d y+\int_{0}^{l} g\left(X_{a} Y_{\tau}-X_{\tau} Y_{a}\right) d u, \\
J^{\prime \prime}(0) & =\iint_{A_{0}} g_{a a} d x d y+\int_{0}^{l} Q d u,
\end{aligned}
$$

where

$$
\begin{aligned}
Q=g[ & \left(X_{\tau \tau} Y_{\tau}-X_{\tau} Y_{\tau \tau}\right)\left(X_{\tau} X_{a}+Y_{\tau} Y_{a}\right)-2\left(X_{a \tau} Y_{\tau}-X_{\tau} Y_{a \tau}\right)\left(X_{\tau} X_{a}+Y_{\tau} Y_{a}\right) \\
& \left.+X_{a a} Y_{\tau}-X_{\tau} Y_{a a}\right]+2\left(X_{a} Y_{\tau}-X_{\tau} Y_{a}\right) g_{a}+\left(X_{a} Y_{\tau}-X_{\tau} Y_{a}\right)^{2}\left(g_{v}+g / \rho\right) .
\end{aligned}
$$

2. Statement of the problem. Suppose we have given an arbitrary, fixed surface

$$
\varphi(x, y, z)=0,
$$

which is of class $C^{\prime \prime}$ and has no singular points for all $x, y, z$ that we wish to consider. Let the surface

$$
z=z(x, y)
$$

be of class $C^{\prime \prime}$ for all $x, y, z$ under consideration, and consider that portion of it which is bounded by its intersection with the surface (15) as indicated in Figure 3. Designate by $C_{0}{ }^{\prime}$ the common line of these surfaces and by $C_{0}$ its projection on the $x y$-plane. Consider now the one-parameter family of surfaces

$$
z=z(x, y)+a \zeta(x, y),
$$

where $\zeta$ is an arbitrary function of $x, y$ which is of class $C^{\prime \prime}$ for all $x, y$ sufficiently near those inside or on $C_{0}$, and where $a$ is a parameter, and where the portions of all surfaces of the family (17) to be considered are those which are bounded by the intersections of these surfaces with the fixed surface (15). These intersections determine a one-parameter family of curves which include $C_{0}{ }^{\prime}$ for $a=0$. The curve of this family with parameter $a$ we call $C_{a}{ }^{\prime}$; its equations are

$\varphi(x, y, z)=0, \quad z=z(x, y)+a \zeta(x, y)$.

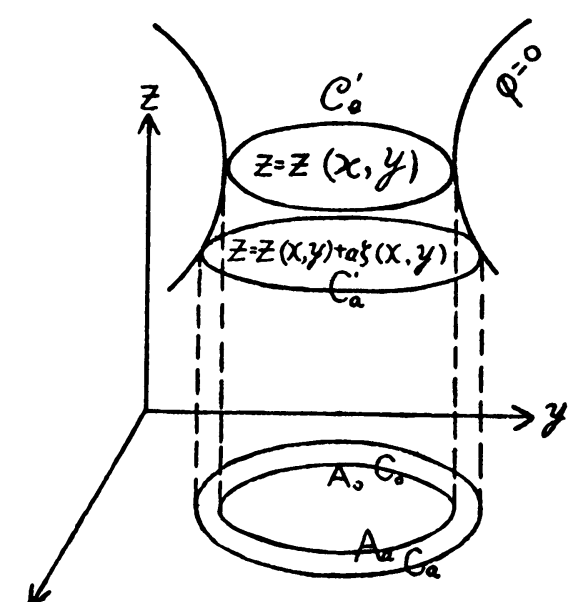

FIG. 3 
Denote by $C_{a}$ the projection of the $x y$-plane of $C_{a}^{\prime}$ and designate by $A_{a}$ the area in the $x y$-plane bounded by $C_{a}$.

Consider now the double integral

$$
I=\iint_{A_{0}} f(x, y, z, p, q) d x d y,
$$

where $A_{0}$ is the area bounded by the curve $C_{0}$ (see Figure 3 ), where $p \equiv z_{x}$, $q \equiv z_{y}$, and where $f$ is a function of class $C^{\prime \prime \prime}$ for all sets $(x, y, z, p, q)$ sufficiently near those on that portion of the surface $z=z(x, y)$ which is bounded by $C_{0}{ }^{\prime}$. We observe that

$$
I(a)=\iint_{A_{a}}\left(x, y, z+a \zeta, p+a \zeta_{x}, q+a \zeta_{y}\right) d x d y
$$

is the value of the integral (19) taken over the portion of the surface (17) which is bounded by its intersection with the surface $\varphi(x, y, z)=0$. Our problem is to obtain the first and second derivatives $I^{\prime}(0)$ and $I^{\prime \prime}(0)$ of this integral in symmetric, usable forms. In doing so, we shall assume that $f \neq 0$ on the surface $z=z(x, y)$ along its intersection $C_{0}{ }^{\prime}$ with the surface $\varphi=0$. This is a customary assumption for problems of the calculus of variations with variable limits, the reason for which will appear later.

3. The first variation. We apply now to the integral (20)

$$
I(a)=\iint_{A_{a}} f\left(x, y, z+a \zeta, p+a \zeta_{x}, q+a \zeta_{y}\right) d x d y
$$

the result in the first of equations (9). Replacing $g$ in that equation by $f$, we obtain

$$
I^{\prime}(0)=\iint_{A_{0}} f_{a} d x d y+\int_{0}^{l} f v_{a} d u,
$$

where $A_{0}$ is as defined in $\$ 2$ (see also Figure 3 ), where

$$
f_{a}=f_{z} \zeta+f_{p} \zeta_{x}+f_{q} \zeta_{y}
$$

and where $v_{a}$ can be computed as follows. Consider the boundary $C_{a}{ }^{\prime}$. It lies on the surface $z=z(x, y)+a \zeta(x, y)$ and on the surface $\varphi(x, y, z)=0$, and when we transform $x, y$ by equation (3), the function $v(u, a)$, which in equations (18) determines the family of curves $C_{a}$, is a solution of the equation

$$
\varphi\left[\xi+v \eta^{\prime}, \eta-v \xi^{\prime}, z\left(\xi+v \eta^{\prime}, \eta-v \xi^{\prime}\right)+a \zeta\left(\xi+v \eta^{\prime}, \eta-v \xi^{\prime}\right)\right]=0
$$


which contains the variables $u, v, a$. Differentiating (23) with respect to $a$, we obtain

$$
v_{a}=-\varphi_{a} / \varphi_{v}
$$

But when $a=0$,

$$
\varphi_{a}=\varphi_{z} \zeta, \quad \varphi_{v}=\left(\varphi_{x}+p \varphi_{z}\right) \eta^{\prime}-\left(\varphi_{y}+q \varphi_{z}\right) \xi^{\prime}
$$

Hence

$$
v_{a}=\frac{-\varphi_{z} \zeta}{\left(\varphi_{x}+p \varphi_{z}\right) \eta^{\prime}-\left(\varphi_{y}+p \varphi_{z}\right) \xi^{\prime}}
$$

The denominator of the right member of (26) is $\varphi_{v}$. It is different from zero; otherwise we should have the two equations

$$
\begin{aligned}
& \left(\varphi_{x}+p \varphi_{z}\right) \eta^{\prime}-\left(\varphi_{y}+q \varphi_{z}\right) \xi^{\prime}=0, \\
& \left(\varphi_{x}+p \varphi_{z}\right) \xi^{\prime}+\left(\varphi_{y}+q \varphi_{z}\right) \eta^{\prime}=0 \quad \text { (by (29)), }
\end{aligned}
$$

and therefore $\varphi_{x}+p \varphi_{z}=\varphi_{y}+q \varphi_{z}=0$, since $\left(\xi^{\prime}, \eta^{\prime}\right) \neq(0,0)$. This would imply that $p: q:-1=-\varphi_{x} / \varphi_{z}:-\varphi_{y} / \varphi_{z}:-1$ and hence that the two surfaces $z=z(x, y)$ and $\varphi(x, y, z)=0$ are tangent to each other. But with $f \neq 0$, which we have supposed, this is impossible, as will be seen in $\S 3$.

Using (22) and (26) in (21), we now obtain the first derivative

$$
I^{\prime}(0)=\iint_{A_{0}}\left(f_{z} \zeta+f_{p} \zeta_{x}+f_{Q} \zeta_{y}\right) d x d y-\int_{0}^{l} \frac{\zeta f \varphi_{z} d u}{\left(\varphi_{x}+p \varphi_{z}\right) \eta^{\prime}-\left(\varphi_{y}+q \varphi_{z}\right) \xi^{\prime}} .
$$

This result we express in the following theorem:

THEOREM 2. The first derivative $I^{\prime}(0)$ of the double integral $I(a)$ of equation (20), taken over the portion of the surface $z=z(x, y)+a \zeta(x, y)$ bounded by its intersection with the surface $\varphi(x, y, z)=0$, has the value given by (27).

In certain applications, it is desirable to change the form of (27) by performing an integration by parts on the double integral and then applying to the result Green's theorem for the plane. Thus we may write

$$
f_{p} \zeta_{x}=\frac{\partial}{\partial x} f_{p} \zeta-\zeta \frac{\partial}{\partial x} f_{p}, f_{q} \zeta_{y}=\frac{\partial}{\partial y} f_{q} \zeta-\zeta \frac{\partial}{\partial y} f_{q}
$$

then the double integral in (27) takes the form

$$
\begin{aligned}
& \iint_{A_{0}}\left[\zeta\left(f_{z}-\frac{\partial}{\partial x} f_{p}-\frac{\partial}{\partial y} f_{q}\right)+\frac{\partial}{\partial x}\left(f_{p} \zeta\right)+\frac{\partial}{\partial y}\left(f_{q} \zeta\right)\right] d x d y \\
& =\iint_{A_{0}} \zeta\left(f_{z}-\frac{\partial}{\partial x} f_{p}-\frac{\partial}{\partial y} f_{q}\right) d x d y+\iint_{A_{0}}\left[\frac{\partial}{\partial x}\left(f_{p} \zeta\right)+\frac{\partial}{\partial y}\left(f_{q} \zeta\right)\right] d x d y .
\end{aligned}
$$


Applying Green's theorem for the plane to the last written integral, we find

$$
\iint_{A_{0}}\left[\frac{\partial}{\partial x}\left(f_{p} \zeta\right)+\frac{\partial}{\partial y}\left(f_{q} \zeta\right)\right] d x d y=\int_{0}^{l} \zeta\left(f_{p} \eta^{\prime}-f_{q} \xi^{\prime}\right) d u
$$

Now substituting in (27) the value found for the double integral, we obtain

$$
\begin{aligned}
I^{\prime}(0)= & \iint_{A_{0}} \zeta\left(f_{z}-\frac{\partial f_{p}}{\partial x}-\frac{\partial f_{q}}{\partial y}\right) d x d y \\
& +\int_{0}^{l} \zeta\left[\frac{\left(f_{p} \eta^{\prime}-f_{q} \xi^{\prime}\right)\left(\left(\varphi_{x}+p \varphi_{z}\right) \eta^{\prime}-\left(\varphi_{y}+q \varphi_{z}\right) \xi^{\prime}\right)-\zeta f \varphi_{z}}{\left(\varphi_{x}+p \varphi_{z}\right) \eta^{\prime}-\left(\varphi_{y}+q \varphi_{z}\right) \xi^{\prime}}\right] d u .
\end{aligned}
$$

Since, along $C_{0}{ }^{\prime}, \varphi[\xi, \eta, \zeta(\xi, \eta)] \equiv 0$ in $u$, we can simplify the line integral of (28). Differentiating this identity, we obtain

$$
\left(\varphi_{x}+p \varphi_{z}\right) \xi^{\prime}+\left(\varphi_{y}+q \varphi_{z}\right) \eta^{\prime}=0 .
$$

Hence from equations (25) and (29), we have

$$
\xi^{\prime}=-\left(\varphi_{y}+q \varphi_{z}\right) / \varphi_{v}, \quad \eta^{\prime}=\left(\varphi_{x}+p \varphi_{z}\right) / \varphi_{v}
$$

where

$$
\varphi_{v}^{2}=\left(\varphi_{x}+p \varphi_{z}\right)^{2}+\left(\varphi_{y}+q \varphi_{z}\right)^{2} .
$$

Substitution from these formulas (30), (31) in the line integral of (28) and application of the relation $\xi^{\prime 2}+\eta^{\prime 2}=1$, reduces (28), after simple computation, to the form

$$
\begin{aligned}
I^{\prime}(0)=\iint_{A_{0}} \zeta & \left(f_{z}-\frac{\partial}{\partial x} f_{p}-\frac{\partial}{\partial y} f_{q}\right) d x d y \\
& +\int_{0}^{l} \zeta\left[\frac{f_{p}\left(\varphi_{x}+p \varphi_{z}\right)+f_{q}\left(\varphi_{y}+q \varphi_{z}\right)-f \varphi_{z}}{\left(\varphi_{x}+p \varphi_{z}\right) \eta^{\prime}-\left(\varphi_{y}+q \varphi_{z}\right) \xi^{\prime}}\right] d u .
\end{aligned}
$$

We therefore have the following corollary to Theorem 2.

Corollary 1. The first derivative $I^{\prime}(a)$ of the double integral $I(a)$, of equation (20), taken over the portion of the surface $z=z(x, y)+a \zeta(x, y)$ bounded by its intersection with the surface $\varphi(x, y, z)=0$, has the value given by (32).

In case $z=z(x, y)$ is a minimizing surface for the integral $I, I^{\prime}(0)$ must vanish. But it is from this result (32) that the Euler necessary condition for double integrals with fixed limits, namely

$$
f_{z}-\frac{\partial}{\partial x} f_{p} \zeta-\frac{\partial}{\partial y} f_{q} \zeta=0,
$$

is obtained by the usual proof* and this condition is of course necessary

* For example, see Bolza's Vorlesungen über Variationsrechnung, p. 655. 
for a minimum in the case of variable limits. When the double integral (32) vanishes, the line integral there must also vanish for every $\zeta(x, y)$. By a slight modification of the fundamental lemma for double integrals, we therefore have further the transversality condition*

$$
f_{p} \varphi_{x}+f_{q} \varphi_{y}+\left(p f_{p}+q f_{q}-f\right) \varphi_{z}=0 .
$$

Hence

COROLlaRy 2. In case $z=z(x, y)$ is a minimizing surface for the double integral

$$
I=\iint_{A_{0}} f(x, y, z, p, q) d x d y,
$$

the Euler equation (33) must hold at every point of the portion of the surface $z=z(x, y)$ inside $C_{0^{\prime}}$, and the transversality condition (34) must hold at every point of the boundary $C_{0}{ }^{\prime}$, which is the line of intersection of the surfaces $z=z(x, y)$ and $\varphi(x, y, z)=0$.

From the hypothesis made in $\$ 2$ that $f \neq 0$ along $C_{0}{ }^{\prime}$, it follows that the surface $z=z(x, y)$ cannot be tangent to the surface $\varphi(x, y, z)=0$ at any point of their intersection $C_{0}{ }^{\prime}$.

For the special case of the minimal surface, $f=\sqrt{1+p^{2}+q^{2}}$ and the transversality condition (34) reduces to

$$
p \varphi_{x}+q \varphi_{y}-\varphi_{z}=0,
$$

which shows that the surfaces $z=z(x, y)$ and $\varphi(x, y, z)=0$ are orthogonal to each other.

4. The second variation. To get $I^{\prime \prime}(0)$, we apply to the integral (20),

$$
I(a)=\iint_{A_{a}} f\left(x, y, z+a \zeta, p+a \zeta_{x}, q+a \zeta_{y}\right) d x d y,
$$

the result obtained in the second of equations (9), $\$ 1$. Replacing $g$ in that equation by $f$, we obtain

$$
I^{\prime \prime}(0)=\iint_{A_{0}} f_{a a} d x d y+\int_{0}^{l} L d u,
$$

where $L=f\left(v_{a a}+v_{a}^{2} / \rho\right)+f_{v} v_{a}^{2}+2 f_{a} v_{a}$. The function $v_{a}$ of course has the value given by (26), and $v_{a a}$ can be computed by differentiating equation (24), $\varphi_{v} v_{a}+\varphi_{a}=0$, with respect to $a$. The result of this differentiation is

$$
v_{a a}=-\frac{1}{\varphi_{v}}\left(\varphi_{v v} v_{a}^{2}+2 \varphi_{a v} v_{a}+\varphi_{a a}\right) .
$$

\footnotetext{
* See Bolza, loc. cit., p. 671.
} 
Carrying out the differentiation indicated in (36) and remembering that the arguments in $\varphi$ are

$$
\xi+v \eta^{\prime}, \quad \eta-v \xi^{\prime}, \quad z\left(\xi+v \eta^{\prime}, \eta-v \xi^{\prime}\right)+a \xi\left(\xi+v \eta^{\prime}, \eta-v \xi^{\prime}\right),
$$

we obtain after collecting terms suitably

$$
\begin{aligned}
& v_{a a}=-\frac{1}{\varphi_{v}^{3}}\left\{\varphi _ { z } ^ { 2 } \zeta ^ { 2 } \left[\varphi_{x x} \eta^{\prime 2}+\varphi_{y y} \xi^{\prime 2}+\varphi_{z z}\left(p \eta^{\prime}-q \xi^{\prime}\right)^{2}-2 \varphi_{x y} \xi^{\prime} \eta^{\prime}\right.\right. \\
& \left.+2 \varphi_{x z} \eta^{\prime}\left(p \eta^{\prime}-q \xi^{\prime}\right)-2 \varphi_{y z} \xi^{\prime}\left(p \eta^{\prime}-q \xi^{\prime}\right)+\varphi_{z}\left(r \eta^{\prime 2}-2 s \xi^{\prime} \eta^{\prime}+t \xi^{\prime 2}\right)\right] \\
& \left.-2 \varphi_{z} \zeta \varphi_{v}\left[\varphi_{x z} \eta^{\prime} \zeta-\varphi_{y z} \xi^{\prime} \zeta+\varphi_{z z}\left(p \eta^{\prime}-q \xi^{\prime}\right) \zeta+\varphi_{z}\left(\zeta_{x} \eta^{\prime}-\zeta_{y} \xi^{\prime}\right)\right]+\left[\varphi_{z z} \zeta^{2} \varphi_{v}^{2}\right]\right\}
\end{aligned}
$$

where, according to custom, $r, s, t$ stand for the derivatives $r=z_{x x}, s=z_{x y}$, $t=z_{y y}$, and where $\varphi_{v} \neq 0$, as was explained just after equation (26). But on collecting the coefficients of $\xi^{\prime 2}, \eta^{\prime 2}$, and $\xi^{\prime} \eta^{\prime}$ in (37), we find for $v_{a a}$ the value

$$
v_{a a}=\frac{\zeta^{2}}{\Delta^{3}}\left[\left(r_{1}-r\right) \eta^{\prime 2}-2\left(s_{1}-s\right) \xi^{\prime} \eta^{\prime}+\left(t_{1}-t\right) \xi^{\prime 2}\right]+\frac{2 \zeta}{\Delta^{3}}\left[\zeta_{x}\left(p-p_{1}\right)+\zeta_{y}\left(q-q_{1}\right)\right],
$$

where

$$
\begin{aligned}
& \Delta=\sqrt{\left(p-p_{1}\right)^{2}+\left(q-q_{1}\right)^{2}}, \quad p_{1}=-\varphi_{x} / \varphi_{z}, \quad q_{1}=-\varphi_{y} / \varphi_{z}, \\
& r_{1}=\partial p_{1} / \partial x=-\frac{1}{\varphi_{z}^{3}}\left(\varphi_{x x} \varphi_{z}^{2}-2 \varphi_{x} \varphi_{z} \varphi_{x z}+\varphi_{x}^{2} \varphi_{z z}\right), \\
& s_{1}=\partial q_{1} / \partial x=-\frac{1}{\varphi_{z}^{3}}\left(\varphi_{x y} \varphi_{z}^{2}-\varphi_{y} \varphi_{z} \varphi_{x z}-\varphi_{x} \varphi_{z} \varphi_{y z}+\varphi_{x} \varphi_{y} \varphi_{z z}\right), \\
& t_{1}=\partial q_{1} / \partial y=-\frac{1}{\varphi_{z}^{3}}\left(\varphi_{y} \varphi_{z}^{2}-2 \varphi_{y} \varphi_{z} \varphi_{y z}+\varphi_{y}^{2} \varphi_{z z}\right) .
\end{aligned}
$$

The introduction of $p_{1}, q_{1}, r_{1}, s_{1}, t_{1}$, thus putting $\varphi_{x}^{3}$ in the denominators of $p_{1}, q_{1}, r_{1}, s_{1}, t_{1}$, seems to require that the surface $\varphi(x, y, z)=0$ shall be representable in the form $z=z_{1}(x, y)$, but in the final form of the integrand of the line integral to be obtained, we shall see that this apparent requirement is not essential. The other three terms of $L$ are $f v_{a}^{2} / \rho$ and the two terms

$$
f_{v} v_{a}^{2}=\frac{\zeta^{2}}{\Delta^{3}}\left[(f)_{x}\left(p-p_{1}\right)+(f)_{y}\left(q-q_{1}\right)\right]
$$

where

$$
2 f_{a} v_{a}=-\frac{2 \zeta^{2}}{\Delta} f_{z}-\frac{2 \zeta}{\Delta}\left(f_{p} \zeta_{x}+f_{q} \zeta_{y}\right)
$$

$$
\begin{aligned}
& (f)_{x}=f_{x}+p f_{z}+r f_{p}+s f_{q}, \\
& (f)_{y}=f_{y}+q f_{z}+s f_{p}+t f_{q} .
\end{aligned}
$$


The terms of $L$ may therefore be collected so as to give the sum $E_{1}+E_{2}$, where

$$
\begin{aligned}
& E_{1} \equiv \frac{\zeta^{2}}{\Delta^{3}}\left[f\left(r_{1}-r\right) \eta^{2}-2 f\left(s_{1}-s\right) \xi^{\prime} \eta^{\prime}+f\left(t_{1}-t\right) \xi^{\prime 2}+\frac{f \Delta}{\rho}+(f)_{x}\left(p-p_{1}\right)\right. \\
& \left.\quad+(f)_{y}\left(q-q_{1}\right)-2 f_{z} \Delta^{2}\right]
\end{aligned}
$$

At first thought one might think that the expression $E_{1}+E_{2}$, copied from (42), is the simplest form obtainable for $L$. We next show that this is not the case. In fact, we shall eliminate from $L$ the derivatives $\zeta_{x}$ and $\zeta_{y}$, which occur only in $E_{2}$.

In $E_{2}$ use $f=f_{p}\left(p-p_{1}\right)+f_{q}\left(q-q_{1}\right)$, an immediate consequence of the transversality condition (34). Then, upon multiplying out the products in $E_{2}$ and re-collecting its terms suitably, we find

$$
E_{2}=2\left(f_{p} \xi^{\prime}+f_{q} \eta^{\prime}\right)\left(\zeta_{x} \xi^{\prime}+\zeta_{y} \eta^{\prime}\right) v_{a}
$$

where we are to observe from (30) and (26) that

$$
\xi^{\prime}=-\left(q-q_{1}\right) / \Delta, \quad \eta^{\prime}=\left(p-p_{1}\right) / \Delta, \quad v_{a}=-\zeta / \Delta .
$$

Fortunately, the factor $\zeta_{x} \xi^{\prime}+\zeta_{\nu} \eta^{\prime}$ of $E_{2}$, in (43), occurs in the formula for $v_{a}{ }^{\prime}$ found from the solution $v(u, a)$ of $\varphi=0$ (see equation (23)). Indeed one readily finds that the equation defining $v_{a}{ }^{\prime}$ is

$$
\zeta_{x} \xi^{\prime}+\zeta_{y} \eta^{\prime}=\left[\left(r_{1}-r\right)-\left(t_{1}-t\right)\right] \xi^{\prime} \eta^{\prime} v_{a}+\left(s_{1}-s\right)\left(\eta^{\prime 2}-\xi^{\prime 2}\right) v_{a}-v_{a}{ }^{\prime} \Delta
$$

after neglecting a term which has $\xi^{\prime} \xi^{\prime \prime}+\eta^{\prime} \eta^{\prime \prime}=0$ as a factor. Using in (43) the value of $\zeta_{x} \xi^{\prime}+\zeta_{\nu} \eta^{\prime}$ which (44) gives, we obtain a second new form of $E_{2}$, namely,

$$
\begin{gathered}
E_{2}=2\left(f_{p} \xi^{\prime}+f_{q} \eta^{\prime}\right) v_{a}\left[\left(r_{1}-r\right)-\left(t_{1}-t\right)\right] \xi^{\prime} \eta^{\prime} v_{a}+\left(s_{1}-s\right)\left(\eta^{\prime 2}-\xi^{\prime 2}\right) v_{a} \\
-2 v_{a} v_{a}^{\prime} \Delta\left(f_{p} \xi^{\prime}+f_{q} \eta^{\prime}\right) .
\end{gathered}
$$

The only part of $E_{2}$ which involves $\zeta_{x}$ and $\zeta_{y}$ is $v_{a}^{\prime}$, which we eliminate presently. Let $S \equiv \Delta\left(f_{p} \xi^{\prime}+f_{q} \eta^{\prime}\right)$, so that the last term of $E_{2}$ in (45) is $-S \cdot 2 v_{a} v_{a}{ }^{\prime}$. Since this term is in $L$, let us integrate it by parts. We have

$$
\left.-\int_{0}^{l} S \cdot 2 v_{a} v_{a}^{\prime} d u=-S v_{a}^{2}\right]_{0}^{l}+\int_{0}^{l} v_{a}^{2} S^{\prime} d u,
$$

where the first term on the right in (46) vanishes since $S v_{a}^{2}$ has the period $l$, and where, after using the relations $\xi^{\prime \prime}=-\eta^{\prime} / \rho, \eta^{\prime \prime}=\xi^{\prime} / \rho$, we find that

$$
\begin{gathered}
S^{\prime}=\left[-f_{p} \eta^{\prime} / \rho+f_{q} \xi^{\prime} / \rho+\xi^{\prime 2}\left(f_{p}\right)_{x}+\eta^{\prime 2}\left(f_{q}\right)_{y}+\left(\left(f_{p}\right)_{y}+\left(f_{q}\right)_{x}\right) \xi^{\prime} \eta^{\prime}\right] \Delta \\
+\left[\left(r-r_{1}\right)-\left(t-t_{1}\right)\right] \xi^{\prime} \eta^{\prime}+\left(s-s_{1}\right)\left(\eta^{\prime 2}-\xi^{\prime 2}\right) .
\end{gathered}
$$


The integration by parts thus gives us the result that we may replace the last term of $E_{2}$ in (45) by $v_{a}^{2} S^{\prime}$, where $S^{\prime}$ is defined by (47). Hence we may replace $E_{2}$ in $L$ by

$$
\begin{aligned}
E= & \left(f_{p} \xi^{\prime}+f_{q} \eta^{\prime}\right) v_{a}\left[\left(r_{1}-r\right)-\left(t_{1}-t\right)\right] \xi^{\prime} \eta^{\prime} v_{a}+\left(s_{1}-s\right)\left(\eta^{\prime 2}-\xi^{\prime 2}\right) v_{a} \\
& +v_{a}^{2} \Delta\left[\left(f_{p}\right)_{x} \xi^{\prime 2}+\left(f_{q}\right)_{y} \eta^{\prime 2}+\left(\left(f_{p}\right)_{y}+\left(f_{q}\right)_{x}\right) \xi^{\prime} \eta^{\prime}\right]+v_{a}^{2} \Delta\left(-f_{p} \eta^{\prime} / \rho+f_{q} \xi^{\prime} / \rho\right),
\end{aligned}
$$

where $v_{a}^{2} \Delta\left(-f_{p} \eta^{\prime}+f_{q} \xi^{\prime}\right) / \rho=-f v_{a}^{2} / \rho$, as is seen immediately from formulas (30) and (34). But $-f v_{a}^{2} / \rho$ exactly cancels with $f \zeta^{2} \Delta / \rho \Delta^{3}=f v_{a}^{2} / \rho$ in $E_{1}$ (see equation (42)). By collecting the remaining terms of $E_{1}$ and $E$, after using the values of $\xi^{\prime}, \eta^{\prime}$, and $v_{a}$ written just below equation (43), and after putting $f=f_{p}\left(p-p_{1}\right)+f_{q}\left(q-q_{1}\right)$ in $E_{1}$, and also after neglecting a term equal to the Euler expression (see equation (33)), we obtain the following form for $I^{\prime \prime}(0)$ :

$$
I^{\prime \prime}(0)=\iint_{A_{0}} 2 \Omega d x d y+\int_{0}^{l} \zeta^{2}\left(A_{1}+A_{2}\right) d u,
$$

where

$$
\begin{aligned}
f_{a a} & \equiv 2 \Omega \equiv f_{z z} \zeta^{2}+2 f_{p z} \zeta \zeta_{x}+f_{p p} \zeta_{x}^{2}+2 f_{p_{q}} \zeta_{x} \zeta_{y}+2 f_{q z} \zeta \zeta_{\nu}+f_{q q} \zeta_{\nu}^{2}, \\
A_{1} & \equiv \frac{1}{\Delta^{3}}\left[f_{p}\left(p-p_{1}\right) r_{1}+\left(f_{p}\left(q-q_{1}\right)+f_{q}\left(p-p_{1}\right)\right) s_{1}+f_{q}\left(q-q_{1}\right) t_{1}\right],
\end{aligned}
$$

$$
\begin{aligned}
A_{2} \equiv & -\frac{1}{\Delta^{3}}\left\{\left[f_{p}\left(p-p_{1}\right) r+\left(f_{p}\left(q-q_{1}\right)+f_{q}\left(p-p_{1}\right)\right) s+f_{q}\left(q-q_{1}\right) t\right]\right. \\
& -\left[(f)_{x}\left(p-p_{1}\right)+(f)_{y}\left(q-q_{1}\right)\right] \\
& +\left[\left(f_{p}\right)_{y}+\left(f_{q}\right)_{x}\right]\left(p-p_{1}\right)\left(q-q_{1}\right)+\left(f_{p}\right)_{x}\left(p-p_{1}\right)^{2}+\left(f_{q}\right)_{y}\left(q-q_{1}\right)^{2} \\
& \left.+\left[f_{z}+\left(f_{p}\right)_{x}\right]\left(p-p_{1}\right)^{2}+\left[f_{z}+\left(f_{q}\right)_{y}\right]\left(q-q_{1}\right)^{2}\right\}
\end{aligned}
$$

the meaning of the notations $\left(f_{p}\right)_{x},\left(f_{q}\right)_{x}$, etc. being obvious from equations (41).

Two things are to be noted here. First, the only place where the curvature of the $\varphi$-surface appears in the line integral of (49) is in $A_{1}$, which contains linearly the elements $r_{1}, s_{1}, t_{1}$, and also $p, q$ and $p_{1}, q_{1}$, which define the normals to the $z$ - and $\varphi$-surfaces; while $A_{2}$ involves the curvature of the $z$-surface, $r, s, t$ appearing linearly, and the elements $p, q$ and $p_{1}, q_{1}$. Secondly, if in $A_{1}, A_{2}$ we substitute for $p_{1}, q_{1}, r_{1}, s_{1}, t_{1}$ their values given in equations (39), we observe that $\varphi_{z}$ does not occur in the denominator of either of the resulting expressions for $A_{1}$ and $A_{2}$, in (50). Hence we see that the apparent necessity of making the restriction that $\varphi_{z} \neq 0$ just after equations (39) was not essential. 
Equation (49) states the result which we set out to obtain. We express it in the following theorem.

THEOREM 3. The second derivative $I^{\prime \prime}(0)$ of the double integral $I(a)$ of equation (20), taken over the portion of the surface $z=z(x, y)+a \zeta(x, y)$ bounded by its intersection with the surface $\varphi(x, y, z)=0$, has the value given by the formula (49), where $f_{a a}, A_{1}$, and $A_{2}$ are the expressions defined in equations (50).

5. Boundary value problem associated with the second variation. A new necessary condition in order that the surface $z=z(x, y)$ shall minimize the double integral (19) is easily deducible from equation (49). To obtain this condition, we first perform an integration by parts on the double integral of (49) and then apply Green's theorem to the result. Then according to Euler's theorem on homogeneous functions, we may write

$$
\iint_{\Lambda_{0}} 2 \Omega d x d y=\iint_{A_{0}}\left[\zeta\left(\Omega_{\zeta}-\frac{\partial}{\partial x} \Omega_{\zeta x}-\frac{\partial}{\partial y} \Omega_{\zeta_{y}}\right)+\frac{\partial}{\partial x}\left(\Omega_{\zeta_{x} \zeta}\right)+\frac{\partial}{\partial y}\left(\Omega_{\zeta_{y} \zeta}\right)\right] d x d y
$$

Now applying Green's theorem for the plane to the last two terms of the right member of (51), we obtain

$$
\begin{aligned}
\iint_{A_{0}} 2 \Omega d x d y=\iint_{A_{0}} \zeta\left(\Omega_{\zeta \zeta}\right. & \left.\frac{\partial}{\partial x} \Omega_{\zeta x}-\frac{\partial}{\partial y} \Omega_{\zeta_{y}}\right) d x d y \\
& +\int_{0}^{l} \zeta\left(\Omega_{\zeta x} \eta^{\prime}-\Omega_{\zeta y} \xi^{\prime}\right) d u,
\end{aligned}
$$

since $u$ is the arc on $C_{0}$. Taking the three equations (49), (50), and (52) into account, we may now write

$$
I^{\prime \prime}(0)=\iint_{A_{0}} \zeta \Psi(\zeta) d x d y+\int_{0}^{l} \zeta\left(P \zeta+Q \zeta_{x}+R \zeta_{y}\right) d u,
$$

where

$$
\begin{aligned}
\Psi(\zeta) & \equiv \Omega_{\zeta}-\frac{\partial}{\partial x} \Omega_{\zeta x}-\frac{\partial}{\partial y} \Omega_{\zeta y}, \\
P & \equiv A_{1}+A_{2}+\left[f_{p_{z}}\left(p-p_{1}\right)+f_{q z}\left(q-q_{1}\right)\right] \frac{1}{\Delta}, \\
Q & \equiv\left[f_{p p}\left(p-p_{1}\right)+f_{p q}\left(q-q_{1}\right)\right] \frac{1}{\Delta}, \\
R & \equiv\left[f_{p q}\left(p-p_{1}\right)+f_{q q}\left(q-q_{1}\right)\right] \frac{1}{\Delta}
\end{aligned}
$$

$\Delta$ being defined in equation (39).

From (53) we can now state the new necessary condition in order that the surface $z=z(x, y)$ shall minimize the double integral (19): 
THEOREM 4. In order that the surface $z=z(x, y)$ shall minimize the double integral (19) it is necessary that for negative values of $\lambda$ the boundary value problem

$$
\begin{array}{ll}
\Psi(\zeta)-\lambda \zeta=0 & \text { in the region } A_{0}, \\
P \zeta+Q \zeta_{x}+R \zeta_{y}=0 & \text { on the arc } C_{0}
\end{array}
$$

have no solution except $\zeta \equiv 0$, the functions $P, Q, R$ being defined in equations (54).

Theorem 4 may be regarded as an analogue of Hilbert's statement of the Jacobi condition for the simplest problem of the calculus of variations in the plane.

The proof that the boundary value problem (55) has no solution except $\zeta \equiv 0$ is seen by observing that the line integral is 0 for every $\zeta$ that satisfies the second condition of $(55)$ and that a $\zeta$ which satisfies both conditions of (55) gives the double integral in equation (53) the value

$$
\lambda \iint_{A_{0}} \zeta^{2} d x d y .
$$

Now if $\lambda<0, I^{\prime \prime}(0)<0$, since the double integral is positive, and hence the second variation $=\epsilon^{2} I^{\prime \prime}(0)$ is negative. Therefore the double integral $I$ of equation (19) is not minimized.

6. The minimal surface. If the surface $z=z(x, y)$ is a surface of minimal area, then the integrand of $I$ is the function $f=\sqrt{1+p^{2}+q^{2}}$. The only derivatives of $f$ which are not zero and which appear in $P, Q, R$ in this case are

$$
\begin{aligned}
f_{p} & =p / f, f_{q}=q / f, f_{p p}=\left(1+q^{2}\right) / f^{3}, f_{p q}=-p q / f^{3}, f_{Q q}=\left(1+p^{2}\right) / f^{3} . \\
(f)_{x} & =(p r+q s) / f, \quad(f)_{y}=(p s+q t) / f, \\
\left(f_{p}\right)_{x} & =\left[\left(1+q^{2}\right) r-p q s\right] / f^{3}, \quad\left(f_{p}\right)_{y}=\left[\left(1+q^{2}\right) s-p q t\right] / f^{3}, \\
\left(f_{q}\right)_{x} & =\left[-p q r+\left(1+p^{2}\right) s\right] / f^{3}, \quad\left(f_{q}\right)_{y}=\left[-p q s+\left(1+p^{2}\right) t\right] / f^{3} .
\end{aligned}
$$

Using these equations (56) we therefore find for $P, Q, R$ the values

$$
\begin{aligned}
P \equiv & {\left[r_{1} p\left(p-p_{1}\right)+s_{1}\left(2 p q-p q_{1}-p_{1} q\right)+t_{1} q\left(q-q_{1}\right)\right] \frac{1}{f \Delta^{3}} } \\
& -\left[r\left(2\left(1+q^{2}\right)\left(p-p_{1}\right)^{2}-p q\left(p-p_{1}\right)\left(q-q_{1}\right)\right)+t\left(2\left(1+p^{2}\right)\left(q-q_{1}\right)^{2}\right.\right. \\
& \left.\left.-p q\left(p-p_{1}\right)\left(q-q_{1}\right)\right)+s\left(\left(2+p^{2}+q^{2}\right)\left(p-p_{1}\right)\left(q-q_{1}\right)-2 p q \Delta^{2}\right)\right] \frac{1}{f^{3} \Delta^{3}}, \\
Q \equiv & {\left[\left(1+q^{2}\right)\left(p-p_{1}\right)-p q\left(q-q_{1}\right)\right] \frac{1}{f^{3} \Delta}, } \\
R \equiv & {\left[-p q\left(p-p_{1}\right)+\left(1+p^{2}\right)\left(q-q_{1}\right)\right] \frac{1}{f^{3} \Delta} . }
\end{aligned}
$$


Since $f_{p z}=f_{q z}=0$, in this case, $P$ has the value which $A_{1}+A_{2}$ assumes for the minimal surface. Hence we have the following corollary to Theorem 3.

Corollary. For the case of the minimal surface, the integral $I^{\prime \prime}(0)$ of equation (49) takes the form

$$
I^{\prime \prime}(0)=\iint_{A_{0}}\left[\left(1+q^{2}\right) \zeta_{x}^{2}-2 p q \zeta_{x} \zeta_{y}+\left(1+p^{2}\right) \zeta_{y}^{2}\right] \frac{1}{f^{3}} d x d y+\int_{0}^{l} P \zeta^{2} d u,
$$

where, in this case, $P=A_{1}+A_{2}$ is defined in equation (57).

Since we also have special values $Q, R$ (see equations (54)) in this case, we may state the following corollary to Theorem 4 .

COROLLARY. In order that the surface of minimum area shall minimize the double integral (19) it is necessary that for negative values of $\lambda$ the boundary value problem

$$
\begin{gathered}
-\frac{\partial}{\partial x} \Omega_{\zeta_{x}}-\frac{\partial}{\partial y} \Omega_{\zeta_{y}}-\lambda \zeta=0 \quad \text { in the region } A_{0}, \\
P \zeta+Q \zeta_{x}+R \zeta_{y}=0 \quad \text { on the arc } C_{0}
\end{gathered}
$$

have no solution except $\zeta \equiv 0$, where the functions $P, Q, R$ are now defined by equations (57) and where

$$
\Omega_{\zeta x}=\frac{\left(1+q^{2}\right) \zeta_{x}-p q \zeta_{y}}{\left(1+p^{2}+q^{2}\right)^{3 / 2}}, \quad \Omega_{\zeta_{y}}=\frac{-p q \zeta_{x}+\left(1+p^{2}\right) \zeta_{y}}{\left(1+p^{2}+q^{2}\right)^{3 / 2}} .
$$

University of Chicago,

Chicago, Ill. 